\title{
Considerations for the Use of Biologic Agents in Patients With Chronic Rhinosinusitis With Nasal Polyposis
}

\author{
Do Hyun Kim (1D · Sung Won Kim (iD) \\ Department of Otolaryngology-Head and Neck Surgery, Seoul St. Mary's Hospital, College of Medicine, \\ The Catholic University of Korea, Seoul, Korea
}

The prevalence of chronic rhinosinusitis with nasal polyps (CRSwNP) is approximately $4.2 \%$ in the United States, $2.1 \%-$ $4.3 \%$ in Europe, and $2.1 \%-8.4 \%$ in Asia. CRSwNP has a worse prognosis than chronic rhinosinusitis without nasal polyps because it is refractory to treatment and relapses are common. Depending on symptom severity, intranasal corticosteroids have been safely and effectively used to treat CRSwNP. However, inconsistent and inadequate delivery to the target site may reduce their effectiveness, necessitating the use of systemic corticosteroids or surgery. Studies have reported polyp recurrence rates ranging from $40 \%$ to $60 \%$ even after surgery because the underlying cause is not addressed [1]. Various monoclonal antibodies that may reduce the underlying type 2 inflammation, such as anti-immunoglobin (Ig) E (omalizumab), anti-interleukin (IL)-4/ IL-13 (dupilumab), anti-IL-5 (mepolizumab, reslizumab), and anti-IL-5 receptor- $\alpha$ (benralizumab), are commercially available [2]. These biologic agents specifically target the overexpressed component of the immune response in CRSwNP, eliminating the need for systemic corticosteroids, which may cause various adverse effects due to generalized immunosuppression and hormonal disturbances. However, various factors need to be considered in the clinical application of biologics.

Patient selection is important because several studies have demonstrated that the CRSwNP type 2 inflammation endotype is predominant in Europe and the United States, but not in Asia [3-5]. Furthermore, studies of asthmatic and atopic dermatitis patients treated using biologics demonstrated varying individual responses and prognoses based on type 2 inflammatory cytokine analysis. The European Position Paper on Rhinosinusitis and Chronic Rhinosinusitis (updated 2020) suggested the following criteria for the application of biologics in CRSwNP ( $\geq 3$ of the following): evidence of type 2 inflammation (tissue eosinophils $\geq 10 /$ high-power field, blood eosinophils $\geq 250 / \mu \mathrm{L}$, or total IgE $\geq 100 \mathrm{IU} / \mathrm{mL}$ ), the need for systemic corticosteroids (systemic steroid courses of $\geq 2$ cycles/year or long-term use $>3$ months) or a contraindication to systemic steroids, a Sino-Nasal Outcome Test score $\geq 40$, a diagnosis of anosmia, and comorbid asthma [6]. An evaluation of clinical traits (bilateral CRSwNP, allergy, asthma, response to systemic corticosteroid therapy, and nonsteroidal anti-inflammatory drug-exacerbated respiratory disease) and serum biomarkers (blood eosinophilia, high or polyclonal serum IgE, and serum Staphylococcus aureus enterotoxin-specific IgE) might be performed for patient selection [7]. From a conservative standpoint, biologic therapies can be considered when there is clear evidence of type 2 inflammation, drug discontinuation causes relapse, or medical and surgical treatments have been ineffective.

Another consideration is the limited information about the potential risks of biologic therapy. In early clinical trials of dupilumab in atopic dermatitis patients, the main adverse effects reported were nasopharyngitis, disease exacerbation, headaches, and injection site problems. However, subsequent studies demonstrated that conjunctivitis ( $8 \%$ ) was the most common adverse effect [8]. Conjunctivitis was reported in $38 \%$ of patients in a subsequent post-marketing analysis, and $11 \%$ of the patients discontinued the drug because of the adverse effects [9]. Although it is difficult to establish a causal relationship because of the small sample size, diseases associated with eosinophilia (transient hypereosinophilia, eosinophilic granulomatosis with polyangiitis, and eosinophilic arthritis) and thromboembolic events have also been reported. In summary, these drugs may affect the entire immune system, causing adverse effects that may only be identified in long-term post-marketing analyses.

An important consideration is the duration of biologic thera-

Copyright (C) 2021 by Korean Society of Otorhinolaryngology-Head and Neck Surgery.

This is an open-access article distributed under the terms of the Creative Commons Attribution Non-Commercial License (https://creativecommons.org/licenses/by-nc/4.0)

which permits unrestricted non-commercial use, distribution, and reproduction in any medium, provided the original work is properly cited. 
py, which is directly related to its cost-effectiveness. The discontinuation of biologics has resulted in clinical signs of polyp recurrence in some cases [10]. These cases suggest that the natural history of CRSwNP may not change in response to biologics, and long-term treatment may be required. Further research is needed to determine the duration of the disease-free period and the recurrence rate after the drug is discontinued. The duration of therapy also affects the cost-effectiveness. As an example, the estimated cost for dupilumab, the first Food and Drug Administration-approved biologic drug, was about USD 36,000 when administered every 4 weeks for 1 year.

The role of biologic agents will evolve as we further investigate the immunological pathophysiology of CRSwNP and its endotypes. Through these efforts, it will be possible to develop a personalized approach for cases of intractable CRSwNP.

\section{CONFLICT OF INTEREST}

No potential conflict of interest relevant to this article was reported.

\section{ORCID}

Do Hyun Kim https://orcid.org/0000-0002-9248-5572

Sung Won Kim https://orcid.org/0000-0002-8981-2536

\section{AUTHOR CONTRIBUTIONS}

Conceptualization: all authors. Writing-original draft: DHK. Writing-review \& editing: SWK.

\section{REFERENCES}

1. DeCondeAS, Mace JC, Levy JM, Rudmik L,Alt JA, Smith TL. Prevalence of polyp recurrence after endoscopic sinus surgery for chronic rhinosinusitis with nasal polyposis. Laryngoscope. 2017 Mar;127(3): 550-5.

2. Franzese CB. The role of biologics in the treatment of nasal polyps. Immunol Allergy Clin North Am. 2020 May;40(2):295-302.

3. Kim DW. Can neutrophils be a cellular biomarker in Asian chronic rhinosinusitis? Clin Exp Otorhinolaryngol. 2019 Nov;12(4):325-6.

4. Wang H, Pan L, Liu Z. Neutrophils as a protagonist and target in chronic rhinosinusitis. Clin Exp Otorhinolaryngol. 2019 Nov;12(4): 337-47.

5. Wang X, Zhang N, Bo M, Holtappels G, Zheng M, Lou H, et al. Diversity of TH cytokine profiles in patients with chronic rhinosinusitis: a multicenter study in Europe, Asia, and Oceania. J Allergy Clin Immunol. 2016 Nov;138(5):1344-53.

6. Fokkens WJ, Lund VJ, Hopkins C, Hellings PW, Kern R, Reitsma S, et al. European position paper on rhinosinusitis and nasal polyps 2020. Rhinology. 2020 Feb;58(Suppl S29):1-464.

7. Cardell LO, Stjarne P, Jonstam K, Bachert C. Endotypes of chronic rhinosinusitis: impact on management. J Allergy Clin Immunol. 2020 Mar;145(3):752-6.

8. Ou Z, Chen C, Chen A, Yang Y, ZhouW.Adverse events of Dupilumab in adults with moderate-to-severe atopic dermatitis: a meta-analysis. Int Immunopharmacol. 2018 Jan;54:303-10.

9. Faiz S, Giovannelli J, Podevin C, Jachiet M, Bouaziz JD, Reguiai Z, et al. Effectiveness and safety of dupilumab for the treatment of atopic dermatitis in a real-life French multicenter adult cohort. J Am Acad Dermatol. 2019 Jul;81(1):143-51.

10. Bachert C, Han JK, Desrosiers M, Hellings PW, Amin N, Lee SE, et al. Efficacy and safety of dupilumab in patients with severe chronic rhinosinusitis with nasal polyps (LIBERTY NP SINUS-24 and LIBERTY NP SINUS-52): results from two multicentre, randomised, double-blind, placebo-controlled, parallel-group phase 3 trials. Lancet. 2019 Nov;394(10209):1638-50. 